\title{
Visibilidad y tendencias teóricas de la educación para la salud en cuatro países de América Latina, 2003-2013
}

\author{
Visibility and Theoretical Trends in the Education for Health in Four Latin-American Countries, 2003-2013 \\ Visibilidade e tendências teóricas da educação para a saúde em quatro países de América Latina, 2003-2013
}

Jennifer Marcela López Ríos ${ }^{a}$

Universidad de Antioquia, Colombia

j.marce89@hotmail.com

ORCID: http://orcid.org/0000-0003-3629-9377

Diana Patricia Molina Berrío

Universidad de Antioquia, Colombia

ORCID: http://orcid.org/0000-0002-1352-1089

Fernando Peñaranda Correa

Universidad de Antioquia, Colombia

ORCID: http://orcid.org/0000-0002-8863-5566
DOI: https://doi.org/10.11144/Javeriana.rgps17-35.vtte Redalyc: http://www.redalyc.org/articulo.oa?id=54557477001

Fecha de recepción: 10 Marzo 2017

Fecha de aprobación: 23 Abril 2018 Fecha de publicación: 01 Noviembre 2018

\section{Resumen:}

Objetivo: analizar las investigaciones realizadas en América Latina sobre educación para la salud durante los últimos diez años, identificando los diferentes desarrollos conceptuales propuestos sobre el tema y las críticas que se le formulan. Método: investigación cualitativa mediante un estado del arte; se analizaron cuarenta artículos de Brasil, Colombia, Cuba y México derivados de las búsquedas realizadas en Pubmed, Lilacs, Redalyc, Scielo, Ovid, Science Direct, Oxford Journals y Dialnet. Resultado: la educación para la salud es un campo multifocal, polisémico, con diferentes propósitos y tendencias teóricas. Se encontró que la mayoría de los autores se ocuparon de asumir una postura frente a ella, pero pocos se dieron a la tarea de conceptualizar la educación y la salud. Conclusión: se perciben diferentes visiones sobre educación para la salud; sin embargo, se cuestiona la poca articulación que se vislumbra en los artículos entre la teoría y la práctica en el tema.

Palabras clave: educación en salud, promoción de la salud, educación, América Latina, revisión, base de datos.

\section{Abstract:}

Objective: To analyze the researches conducted in Latin America on education for health during the last ten years, identifying the different conceptual developments proposed about this theme and the criticism made about it. Methods: This is a qualitative research describing the state of the art; forty articles from Brazil, Colombia, Cuba and Mexico, and retrieved from searches in Pubmed, Lilacs, Redalyc, Scielo, Ovid, Science Direct, Oxford Journals, and Dialnet were examined. Results: The education for health is a multifocal and polysemic field with different purposes and theoretical trends. It was found that most of the authors cared for assuming a stance regarding this field, though few of them undertake the task to conceptualize both education and health. Conclusion: Different visions about education for health were perceived; however, this work criticizes the poor articulation observed in the papers between theory and practice on this regard.

Keywords: education for health, health promotion, education, Latin America, review, database.

\section{Resumo:}

Objetivo: analisar as pesquisas feitas na América Latina sobre educação para a saúde durante os últimos dez anos, a identificar os diferentes desenvolvimentos conceituais propostos sobre o tema e as críticas que lhe são feitas. Método: pesquisa qualitativa através de um estado da arte; analisaram-se quarenta artigos do Brasil, Colômbia, Cuba e México derivados das buscas feitas em Pubmed, Lilacs, Redalyc, Scielo, Ovid, Science Direct, Oxford Journals e Dialnet. Resultado: a educação para a saúde é um campo multifocal, polissémico, com diferentes propósitos e tendências teóricas. Evidenciou-se que a maioria dos autores teve o cuidado de assumir uma posição frente a ela, mas poucos se arrogaram a tarefa de conceituar educação e saúde. Conclusão: diferentes visões

\footnotetext{
a Autor de correspondencia: j.marce89@hotmail.com
} 
sobre educação para a saúde são percebidas, no entanto questiona-se a pouca articulação vislumbrada nos artigos entre a teoria e a prática no tema.

Palavras-chave: educação em saúde, promoção da saúde, educação, América Latina, revisão, base de dados.

\section{Introducción}

La educación para la salud $(\mathrm{EpS})$ ha sido considerada tradicionalmente una dimensión importante dentro de la salud pública; sin embargo, su desarrollo no ha estado acorde a este ideal (1, 2). Por ello, entidades como la Organización Mundial de la Salud (OMS) y la Organización Panamericana de la Salud (OPS) han cuestionado sus resultados, teniendo como base el modelo actual de la salud que es morbicéntrico y la concepción de salud que sigue orientada a la enfermedad. En este sentido, autores como Nutbeam manifiestan que la EpS ha perdido importancia en el campo de la promoción de la salud, debido a que se ha reducido, principalmente, a campañas de corto plazo que buscan cambios de comportamiento individual (2). Por su parte, Serrano y Alzate afirman que existe una debilidad teórica respecto de los fundamentos pedagógicos que orientan la $\operatorname{EpS}(1,3)$. Asimismo, se encuentra escaso debate sobre la concepción de salud que sustentan las propuestas y las acciones en $\operatorname{EpS}(1,4)$.

De otro lado, en investigaciones realizadas en el ámbito colombiano $(5,6)$ se ha encontrado que, en la formación superior, en las profesiones del área de la salud, la EpS no recibe la atención que supuestamente debería recibir, dada la importancia que se le atribuye. Además, se identifica una falta de identidad debido a la tendencia de confundirla con otros asuntos tales como atención primaria en salud y promoción de la salud, que en ocasiones se usan de manera indistinta (6).

En la revisión de la literatura, varios han sido los significados que se han asignado a la EpS: desde una herramienta efectiva para promover cambios y estilos de vida saludables en el individuo y la sociedad hasta visiones que se fundamentan en aspectos como las experiencias que tienen los colectivos frente a su salud y su cuidado.

Igualmente, se reconoce la necesidad de asumir una perspectiva transdisciplinar que reconozca el aporte de otras disciplinas como la pedagogía, la psicología, la sociología y la antropología, entre otras. También, se plantea la educación para la salud como un medio para promover y fortalecer la participación comunitaria y su incidencia en la transformación de realidades (7).

De acuerdo con lo anterior, se consideró importante desarrollar el diseño de un estado del arte en EpS que permitiera avanzar, no solo en la búsqueda de referentes y desarrollos que se han tenido al respecto en algunos países de América Latina, sino también en la comprensión de la situación de la EpS en cuanto a su visibilidad y los sustentos teóricos asumidos por los investigadores. Las preguntas que orientaron la investigación fueron: ¿cuáles son los significados de educación, salud y educación para la salud que presentan los investigadores?, ¿qué tanto se investiga sobre educación para la salud?, ¿qué tipo de investigaciones se llevan a cabo?, ¿cuáles son las tendencias teóricas en educación para la salud?

\section{Método}

Se realizó una investigación cualitativa mediante el diseño de un estado del arte en EpS, entendido este como una investigación documental a partir de la cual se recupera y se trasciende reflexivamente en el conocimiento acumulado sobre determinado objeto de estudio para señalar nuevas formas de ver y explicar la cuestión. Asimismo, se considera un estudio sobre la producción existente —investigativa, teórica, metodológicaacerca de determinado tema para develar, desde ella, la dinámica y la lógica presentes en la descripción, explicación o interpretación que del fenómeno en cuestión formulan los teóricos o los investigadores $(8,9)$. 
Dicho panorama del estado actual permite hacer balances prospectivos, reflexivos, sistemáticos y propositivos, los cuales comparten métodos y técnicas desarrolladas en las investigaciones documentales. De ahí que su unidad de análisis esté constituida por documentos escritos, como artículos de revistas, libros e informes de estudio $(10,11)$. En el caso de esta investigación, la unidad de análisis estuvo conformada por los artículos científicos de EpS hallados en las siguientes bases de datos indexadas, especializadas en el área de la salud: Pubmed, Lilacs, Redalyc, Scielo, Ovid, Science Direct, Oxford Journals, Dialnet.

Desde esta perspectiva, se realizó un rastreo inicial de artículos para el periodo 2003-2013 que versaran sobre resultados de investigaciones realizadas en ocho países de América Latina: Brasil, Colombia, Cuba, México, Puerto Rico, Chile, Argentina y Ecuador. Se realizó un proceso de búsqueda avanzada mediante sistema boleano, a través de la combinación de los descriptores validados por los investigadores, como health education, educação em saúde, educación sanitaria y educación para la salud, en los campos de título, palabra clave y abstract. De esta búsqueda se identificaron 246 artículos, pero un análisis preliminar mostró que muchos de estos no tomaban la educación para la salud como un tema central que pudieran aportar a los objetivos de la investigación. Se decidió entonces descartar los artículos que no tuvieran el término educación para la salud en el título, y se identificaron 146, con la siguiente distribución: Brasil: 98, Colombia: 18, Cuba: 11 y México: 7. De Argentina, Puerto Rico, Chile y Ecuador solo se encontraron 12, por lo cual se decidió omitirlos, porque se esperaba hacer algunas comparaciones entre estos.

En el proyecto se desarrollaron las dos fases que comprende la elaboración de un estado del arte: la heurística y la hermenéutica $(12,13)$. En la primera, se acometió la búsqueda y la recopilación de las fuentes de información, y se logró validar los criterios de selección, los términos, las combinaciones y la estrategia de búsqueda. En la segunda fase se abordó la interpretación y el análisis de la información que permitió comprender el fenómeno, mediante la construcción de un sistema categorial, la codificación de los textos y,finalmente, la interpretación de las relaciones encontradas entre las categorías.

Los criterios de inclusión para los artículos científicos fueron los siguientes: (a) artículos de investigaciones, de revisión de tema o de reflexión; (b) artículos cuyo tema central fuera la educación para la salud (health education o educação em saúde); (c) artículos publicados entre el 2003 y el 2013; (d) artículos disponibles en las bases de datos indexadas del estudio; (e) artículos publicados en español, inglés o portugués; y (f) artículos originados en investigaciones que se hubieran realizado en Brasil, México, Cuba o Colombia.

En una matriz bibliográfica elaborada en Excel se recopiló la información de los 134 artículos encontrados, y en el software Atlas Ti, versión 6.0, se realizó la codificación y la posterior categorización. Se construyeron 194 códigos y se agruparon en cinco categorías: concepción de educación, concepción de salud, concepción de educación para la salud, críticas y propuestas respecto a la educación para la salud. En este artículo se presentan los hallazgos respecto a una de ellas: concepción de educación para la salud.

De los 134 artículos se seleccionaron 40, teniendo en cuenta su pertinencia en relación con las preguntas de investigación. En el caso de Colombia se incluyeron todos los artículos encontrados, dado el interés en analizar el desarrollo de EpS en el ámbito nacional. En cuanto a los aspectos éticos, el Comité de Ética de la Facultad Nacional de Salud Pública de la Universidad de Antioquia aprobó la investigación mediante acta N. ${ }^{\circ} 110$ del 2014.

\section{Resultados}

A continuación se presenta una descripción general sobre los artículos analizados; posteriormente, se identifican tres tendencias en relación con las concepciones de EpS encontradas. La estructuración en dichas tendencias se sustenta en las concepciones de educación y salud que subyacen en cada una de ellas. 


\section{Características de los artículos}

Del total de artículos seleccionados para el análisis ( 40 artículos), se encontró que la mayoría desarrollaba investigaciones con enfoque cualitativo (23 artículos), seguidos de los que utilizaban un enfoque de investigación acción-participación ( 6 artículos) y, en menor medida, se encontraron aquellos que estudiaron la EpS desde una perspectiva cuantitativa ( 3 artículos). La mayoría de los autores plantearon como objetivo central la sistematización, análisis, evaluación o implementación de un programa o proyecto de ámbito comunitario, sugiriendo una estrecha relación entre EpS y trabajo comunitario, sobre la base de que Colombia y México fueron los países que más realizaron este tipo de investigación. Otros tipos de investigación utilizados fueron la revisión de fuentes secundarias y las investigaciones que tenían como objetivo central indagar sobre la formación en $\mathrm{EpS}$ de los profesionales del área de la salud, desarrolladas en su mayoría por investigadores brasileños. Fueron menores las experiencias de la EpS en el campo de la clínica (tabla 1).

De las ocho bases de datos indexadas que se revisaron, Scielo y Lilacs son las que más trabajos tienen indexados en ese periodo, con 57 y 53 artículos respectivamente. El idioma portugués es el predominante, con 76 artículos.

\section{TABLA 1}

Tipo de investigación encontrada en la literatura sobre EpS

\begin{tabular}{|c|c|c|}
\hline Tipo de investigación & $\begin{array}{c}\text { Frecuencia } \\
\text { absoluta }\end{array}$ & $\begin{array}{l}\text { Frecuencia } \\
\text { relativa }(\%)\end{array}$ \\
\hline Investigación en institución educativa & 2 & 5 \\
\hline $\begin{array}{l}\text { Investigación en programa o proyecto } \\
\text { comunitario }\end{array}$ & 12 & 30 \\
\hline $\begin{array}{l}\text { Investigación en un programa de servicios } \\
\text { de salud }\end{array}$ & 3 & 7,5 \\
\hline $\begin{array}{l}\text { Investigación en proceso de formación de } \\
\text { profesionales de la salud }\end{array}$ & 6 & 15 \\
\hline Investigación teórica y reflexiva & 4 & 10 \\
\hline $\begin{array}{l}\text { Investigación de fuentes secundarias- } \\
\text { revisión }\end{array}$ & 10 & 25 \\
\hline Otro & 3 & 7,5 \\
\hline Total & 40 & 100 \\
\hline
\end{tabular}




\section{Concepción de educación para la salud}

Se encontró que la EpS es un campo multifocal, polisémico, con diferentes propósitos y diferentes tendencias teóricas, pues se identificaron diversas concepciones y maneras de abordaje. También se destaca que la mayoría de los autores tienden a presentar una definición o una posición hacia la educación para la salud, pero es menos frecuente que se den a la tarea de conceptualizar la educación y la salud y, en caso de hacerlo, algunos parecen presentar una contradicción entre la concepción de EpS que asumen y la manera como la abordan en la práctica.

En este orden de ideas, se proponen tres tendencias sobre las concepciones de la EpS. Una corresponde a lo que puede denominarse posición tradicional, la segunda corresponde a posiciones alternativas y la tercera estaría configurada por una mezcla de las dos anteriores, lo que podría plantearse como una situación paradójica. La presentación de dichas tendencias se basará en las concepciones de educación y salud que subyacen a las concepciones de EpS.

\section{Posición tradicional de la educación para la salud}

De acuerdo con la literatura utilizada para respaldar este estudio, se entiende como modelo tradicional en educación aquel que se caracteriza por un proceso de enseñanza basado en la transmisión de conocimiento y una relación vertical y unidireccional entre el educador y el educando; es decir, el educador es quien tiene el conocimiento - el que moldea - y el educando es moldeado; el papel de este último es receptivo y se limita a obedecer y a memorizar la información, pues en este proceso la memoria es fundamental y su eje central son los contenidos (14).

A partir de esta concepción, se encontraron artículos que expresaban una posición tradicional de la EpS caracterizada por una perspectiva instrumental, al concebirla como herramienta centrada principalmente en la transmisión de información y de conocimientos. En esta medida, para algunos investigadores la educación se concibe como un instrumento útil para el cambio de las personas, de modo que estas adopten estilos de vida más saludables:

La educación junto con la comunicación y la participación social son definidas como herramientas que se utilizan en la promoción de la salud y la prevención de la enfermedad, produciendo cambios en los estilos de vida de las personas. (15)

En cuanto a la educación, esta se concibe como un medio para suministrar información y transmitir conocimientos. Por lo general, se entiende en el marco de una relación unidireccional en la cual el educador - quien posee los conocimientos, en este caso los conocimientos disciplinares biomédicos — los transmite a un educando desprovistos de estos:

La educación es definida como el acto que permite la transmisión de información y conocimientos para la población y profesionales. Estos conceptos se derivan del conocimiento proporcionado por la formación académica [...]. (16)

Respecto del concepto de salud, se encontró que son menos frecuentes los artículos que plantean un análisis sobre esta, y en los casos que hacen un análisis explícito, se encuentra que prima la referencia a la definición de la salud propuesta por la OMS como bienestar físico, social y mental, y no meramente como la ausencia de enfermedad, lo que implica el autocuidado, la promoción de estilos de vida saludables y de habilidades para superar las adversidades de la vida. Aquí la salud está centrada en lo que debe hacer el sujeto, como el autocuidado para estar sano y la responsabilidad de lograr dicha condición y mantenerla.

Así, la EpS queda definida para unos autores como una estrategia, mientras que para otros es un proceso, y para algunos una herramienta que busca, principalmente, influir en el individuo para que adopte estilos de vida saludables, incorpore cambios en sus hábitos o comportamientos y, por ende, pueda prevenir enfermedades al asumir nuevas actitudes y nuevos modos de cuidado en salud: 
La educación en salud es un proceso que promueve cambios de comportamiento, conceptos y actitudes frente a la salud, la enfermedad, el uso de los servicios y refuerza conductas. (17)

La EpS es una estrategia que permite fomentar la promoción de la salud y la prevención, centrada en la valorización de las experiencias personales y colectivas a fin de influir en las actitudes en salud de los individuos. (18)

La EpS es una estrategia que permite formar a la población, generar conciencia colectiva, conocer la transmisión de enfermedades y la manera de prevenirlas. (19)

Es importante señalar que en las concepciones anteriores la EpS se asocia con promoción de la salud y prevención de la enfermedad. Asimismo, se la relaciona con la comunicación, por lo cual también se la identifica como "una estrategia, intervención, medio de comunicación con la población, de resolución de problemas y de modificación de hábitos” (20).

\section{Posiciones alternativas frente a la educación para la salud tradicional}

En contraste con las anteriores perspectivas de la EpS, se identificaron posiciones alternativas que proponen una educación que trasciende la transmisión de los contenidos, pues buscan que el educando sea el protagonista de su proceso de educación porque conciben el conocimiento como una construcción a partir de la participación y del diálogo entre educando y educador. Asimismo, su eje central es el sujeto y no los contenidos, y por esto se orienta a promover su transformación para que contribuya a la emancipación de la sociedad (14).

En este sentido, la educación - para algunos investigadores- es un proceso que está presente en todo momento de la vida y que, por ello, no debe reducirse al nivel informativo que se refiere, básicamente, a una transmisión de datos entre emisor y receptor mediante una relación de carácter jerárquico, sino más bien a un proceso más integral que comprenda la participación de todos los sujetos comprometidos:

Hay que tener presente que el fundamento de la educación está relacionado con el acontecer del acto en la vida cotidiana, la cual a su vez da sentido a la existencia, no así "los conocimientos, la información, ni las verdades transmitidas a través de discursos y consignas [...] La educación involucra un proceso profundo de enseñanza aprendizaje donde se construyen nuevos saberes; no se debe reducir a un nivel informativo que se refiere a una transmisión de datos emisor-receptor con carácter jerárquico. (21)

Para otros, la educación se concibe como transformadora de desigualdades sociales y contribuyente a la emancipación del sujeto, al ser este último partícipe de su propio proceso de educación:

La concepción crítica de educación parte de que es una educación comprometida con la interpretación crítica de la realidad en la perspectiva de la transformación de las desigualdades sociales y de la emancipación de los sujetos copartícipes de la acción educativa. (22)

Otra visión presenta la educación como un proceso en doble vía, en la cual tanto educador como educando aprenden y se transforman, de modo que ambos desarrollan su autonomía y su libertad como sujetos:

La educación basada en la reflexión, en el diálogo y en el intercambio de experiencias entre educador/educando y profesional/ cliente, permite que ambos aprendan juntos, por medio de un proceso de emancipación. (23)

La educación es entonces el proceso por el cual un ser humano, que aprende, es apoyado por otro ser humano, que enseña, en la construcción, ampliación y sostenimiento de su autonomía, por medio del desarrollo de sus competencias. (24)

Además, la educación se concibe como un proceso que proporciona al individuo condiciones para desarrollar una actitud crítica necesaria, tendiente a lograr la transformación de la realidad y su propio desarrollo como ser humano:

La educación debe llevar al educando a una toma de conciencia y actitud crítica en el sentido de obtener cambios de la realidad, debe ser considerada como parte de la calidad de vida y no puede ser segmentada [...] La educación proporciona al individuo condiciones para que el mismo busque, exponga, cuestione, viva, experimente, cree, contribuya, rescate, conquiste su lugar en la sociedad, alcance sus objetivos e ideas y transforme sus sueños en realidad. (24) 
En cuanto a la concepción de salud, se encontraron visiones alternativas que la plantearon como un derecho humano; es decir, aquel que todas las personas merecen sin importar su sexo, raza, religión o estrato socioeconómico, y que contribuye al desarrollo humano.

La salud ha sido definida contemporáneamente como un derecho humano. Derecho humano significa que toda persona lo merece por el mero hecho de ser humano, independientemente de toda otra consideración de sexo o preferencia sexual, raza, nacionalidad, clase social, religión, partido, entre otros. (25)

Las personas eran objetos de intervención del Estado y la salud era considerada más como un deber que como un derecho, ya que la finalidad de las acciones sobre la población estaban guiadas por intereses económicos. (26)

La salud está relacionada con el desarrollo humano y con la construcción social que hace el sujeto. (27)

Es así como en esta tendencia se encontraron concepciones de EpS que la formulan como un contribuyente a la emancipación y a la construcción de personas sensibles y críticas:

La EpS es un conjunto de actividades emancipadoras que buscan construir personas sensibles y conscientes para enfrentar situaciones individuales y/o colectivas que influyan en su calidad de vida. (26)

La EpS es una herramienta que permite al individuo comprender su entorno, brindándole posibilidades de transformación y creación de ideas para el desarrollo de la sociedad. (28)

También hay quienes asumieron la EpS como una dimensión importante de la salud pública, ya que contribuye al desarrollo humano y a la equidad, en la medida en que ayuda a entender los problemas de esta mediante el análisis de los determinantes sociales de la salud $(6,29)$.

En complemento de la visión anterior, la EpS se planteó cómo el primer derecho de la salud: "El primer derecho en salud debe ser el derecho a una educación en salud, que fomente el desarrollo de la consciencia ambiental y salubrista para poder cuidar de la propia salud" (25).

Otros entendieron la EpS como una práctica social que busca fomentar la visión crítica y ampliada de la salud según se desarrolla en la cotidianidad; es decir, que se puede realizar en cualquier momento y espacio de la vida:

La EpS debe ser considerada como una práctica que puede ser desarrollada en cualquier momento de la vida, logrando la interacción de saberes. (30)

La EpS es un medio para desarrollar una visión crítica de los problemas de salud, es una práctica social que está presente en cada instante de la vida. (24)

\section{Más allá de la tensión entre visiones tradicionales y alternativas: una situación paradójica}

Ahora bien, en esta investigación también se encontraron quienes asumían un concepto de educación alternativo y de salud alternativo, pero en las acciones que proponían de EpS asumían una postura tradicional. Así, se identificaron concepciones que relacionan la educación con un proceso de desarrollo humano y social realizado por agentes activos y transformadores de la realidad y, al mismo tiempo, la proponen como estrategia mediática que busca el cambio de comportamientos y la adopción de estilos de vida saludables, dejando entrever que se aborda desde un modelo biomédico:

El proceso educativo se centra en el desarrollo humano individual y social, buscando el empoderamiento de las personas y de las comunidades, con sujetos conscientes de sus derechos, con responsabilidad social y política, como actores protagónicos en el cambio cultural y en cuanto a agentes transformadores de las condiciones de salud. (6)

La EpS se concibe desde una perspectiva centrada en el cambio del comportamiento y la adopción de estilos de vida saludables a partir de la implementación de diversas estrategias de intervención. (6)

Otras de las posturas que mostraron esta situación paradójica fueron aquellas que pretenden trascender al sujeto en la medida en que la EpS se promueve como estrategia para incidir en los determinantes sociales y ambientales, pero las acciones siguen orientadas al suministro de conocimientos y al cambio de comportamientos y actitudes respecto de la salud, la enfermedad y el uso de los servicios de salud (31). 


\section{Discusión}

Esta investigación deja entrever tres hallazgos principales que se describirán a lo largo de este apartado: (1) reducido desarrollo en la investigación sobre $\mathrm{EpS}$ que se manifiesta un reducido número de publicaciones en la temática, (2) identificación de tres tendencias en EpS y, finalmente, (3) situación paradójica entre visiones tradicionales y alternativas.

Un primer acercamiento a la reducida producción en investigación en EpS se podría deber a que esta quede "encubierta" dentro las investigaciones sobre promoción de la salud, en la medida en que para muchos investigadores la EpS es una de sus estrategias. Lo anterior concordaría con el análisis que hacen Arroyo y Cardaci respecto de su preocupación por la manera como la promoción de la salud ha invisibilizado la EpS en las últimas décadas $(32,33)$. Otra explicación podría indicar que, dado el carácter comunitario y práctico de una buena parte de la EpS, la publicación se estaría quedando en la literatura gris, por lo que se pierde su difusión a la comunidad académica.

Es posible que también se deba a la poca trascendencia dada a la EpS en los programas de formación profesional del área de la salud y el escaso desarrollo de grupos y líneas de investigación en la temática encontrado en otros estudios $(5,6,29)$.

Sin embargo - como se mencionó anteriormente-, si bien se identifica un reducido número de publicaciones que versan sobre el tema, llama la atención la existencia de una diferencia marcada entre los países que se analizaron, pues mientras en Brasil se encontraron 98 artículos, en Colombia —el país que le sigue en número de artículos- se encontraron 18. Una razón que podría influir para que se presente esta situación podría ser el tamaño geográfico y poblacional de Brasil, así como su sistema de educación superior y de investigación. Empero, este hecho por sí solo tal vez no explicaría dicha diferencia, por lo cual habría que pensar en otras razones. Entre estas se cuenta la promoción explícita de la EpS en el ámbito de las políticas públicas, tal como lo ha hecho Brasil, lo que se materializa en acciones como la creación de la Coordinación General de Apoyo a la Educación Popular y a la Movilización Social del Departamento de Apoyo y Gestión Participativa de la Secretaría de Gestión Estratégica y Participativa del Ministerio de Salud del Brasil en el 2005 (34, 35). Además, habría que tener en cuenta la influencia que ha tenido en Brasil el movimiento de la Educación Popular en el surgimiento del Sistema Único de Salud (SUS).

Dado que el SUS también emergió como un movimiento popular, recogió valiosos aportes de la Educación Popular para la consolidación de su legado político y teórico (36). Por esta razón, el Ministerio de Salud promovió en el 2007 el Cuaderno en Educación Popular, en el cual presenta la relación de los movimientos de educación popular y del SUS, así como la necesidad de promover la Educación Popular como perspectiva teórica y política para realizar la EpS en el Brasil (36).

El segundo elemento de interés para la discusión gira en torno a las tres tendencias de EpS encontradas en el estudio. Una de ellas - la predominante- corresponde a una perspectiva tradicional de la EpS, específicamente por sus escenarios, metodologías, formas de evaluar, roles de los actores que participan y las demás características propias de su abordaje. Desde esta perspectiva, el educando es un alumno que no cuenta con conocimientos y que, por tanto, asiste a la escuela a que los adultos, en este caso los educadores, sean quienes se lo transmitan (37). Este modelo corresponde a lo que Freire denomina educación bancaria, según la cual "el sujeto de la educación es el educador", el cual conduce al educando en la memorización mecánica de los contenidos (38).

Los educandos son así una suerte de "recipientes" en los que se "deposita" el saber" (38). Bajo este modelo, las actividades educativas están estructuradas según una actitud paternalista para mostrar al educando (o paciente) los principales daños y lesiones que puede sufrir si no asume un comportamiento "correcto". En cuanto a la concepción de salud, esta se explica desde el modelo biomédico con una orientación morbicéntrica, lo que concuerda con otros estudios que han encontrado esta misma situación (39). 
En contraste con la anterior modalidad, se identificó que hay quienes asumen la EpS desde una posición más crítica, centrada en el sujeto. En este sentido, la educación se percibe como proceso que está presente en todo momento de la vida, un proceso de acción-reflexión-acción que permite la transformación de realidades, pensamientos y desigualdades sociales mediante una visión crítica y una construcción autónoma del ser, lo que contribuye a su libertad y emancipación, en consonancia con lo expresado por Freire: "La educación es un encuentro que solidariza la reflexión y la acción de sujetos encauzados hacia el mundo que debe ser transformado y humanizado"(38).

En esta tendencia, el protagonista es el individuo y las actividades están orientadas a contribuir a la emancipación del ser, al desarrollo de la sociedad, al fomento del empoderamiento y a la creación de un sujeto con visión crítica, todo lo cual contribuye a la formación de un ser ético, justo, humano y con visión crítica y solidaria (28). En este caso, la EpS tiene como propósito la promoción del desarrollo humano y, en ese sentido, sus intenciones se centran en la transformación de realidades, la autonomía del sujeto y el alcance de los sueños de cada individuo. Aquí "la educación (está) basada en la reflexión, en el diálogo y en el intercambio de experiencias entre educador/educando y profesional/cliente, permitiendo que ambos aprendan juntos, por medio de un proceso de emancipación" (24).

La presencia de estas dos perspectivas en la EpS la ha descrito desde los años ochenta Obrian (40) y ha quedado documentada recientemente en algunos estudios de Brasil (14, 28).

La última tendencia tiene que ver más con un asunto paradójico, en el sentido de que se propone una concepción de educación problematizadora, mediada por un proceso dialógico y con fines emancipadores; sin embargo, la práctica propuesta se centra en la modificación de hábitos para que se asuman estilos de vida saludables. También se encuentra una paradoja entre perspectivas que proponen una visión alternativa de la salud, pero a la hora de plantear la práctica, esta se orienta desde la enfermedad. Esta situación también ha sido documentada por otros académicos e investigadores.

En los años ochenta, Greene y Simons-Morton (41) se quejaban porque los profesionales del área de la salud se plantean perspectivas amplias sobre la salud, pero a la hora de proponer sus acciones, las dirigen a la prevención o el tratamiento de enfermedades. Gazzineli (42), por su parte, encontró que los educadores en salud muchas veces hablan de corrientes y modelos de educación crítica sin que haya cambios significativos en sus prácticas pedagógicas.

Ante este panorama, es necesario señalar que el paradigma biomédico y su influencia en la EpS siguen conservando su vigencia. A pesar de las críticas que ha recibido y de las nuevas perspectivas teóricas que apuntan a construir un concepto de salud que trascienda lo morbicéntrico, continúa siendo predominante en la comprensión que se asume de la salud y, por ende, en las propuestas de intervención que se diseñan para su atención. Esta situación invita a reflexionar en torno a las posibles razones que determinan la predominancia de dicho paradigma entre los profesionales de la salud. Algunos factores que pueden incidir en el mantenimiento de tal paradigma corresponden a aspectos estructurales, como el modelo económico predominante según el cual la salud se concibe como una mercancía, desconociendo su condición de derecho fundamental para la dignificación de la humanidad (43).

Las políticas públicas diseñadas al servicio del modelo económico imperante y la resistencia que algunos grupos sociales ejercen ante la crítica que se le formula al modelo biomédico - ya que cambiarlo implica ceder el poder - constituyen otros dos factores que tener en cuenta (43). Todo lo anterior conforma un entramado de fuerzas que dificultan el ejercicio de llevar a la realidad perspectivas diferentes sobre la educación y la salud en la EpS. En este sentido, se pronuncia Bourdieu cuando plantea que

Las ciencias sociales deberían ser un elemento obligatorio de toda enseñanza destinada a los futuros profesores [...] Para combatir lo que yo llamo efecto de destino, y la tendencia de los profesores a creer que ellos juzgan a la persona, cuando en verdad juzgan a individuos sociales, habría que darles la conciencia de los mecanismos más elementales de la transmisión cultural. (44) 


\section{Conclusiones}

Dado que la EpS no es homogénea y que requiere entenderse como asunto plural, es necesario reconocer la existencia de un campo en conflicto que necesita un análisis teórico y pedagógico profundo. No ha sido la tendencia predominante abordar este debate desde las disciplinas de la salud, pero este estudio muestra la necesidad de hacerlo ante la complejidad del fenómeno.

En este orden de ideas, la EpS debe asumirse, en el campo de la salud, más allá de sus relaciones con la promoción de la salud y la atención primaria en salud. La EpS requiere una reflexión independiente para analizar sus fundamentos teóricos, epistemológicos, éticos y políticos, como punto de partida para pensarla en los campos de las diferentes disciplinas del área de la salud. Pensar la EpS de esta manera exige reflexionar también sobre los correspondientes fundamentos teóricos, políticos, epistemológicos y éticos de las disciplinas del área de la salud en la medida en que estas también están cruzadas por diversas corrientes y perspectivas.

Así que el fortalecimiento de la EpS pasa por el reconocimiento de su importancia para las disciplinas de la salud, por la pregunta por los modelos de salud (biomédico o centrados en la vida) y por la formación del talento humano.

De otro lado, la transformación en el campo de la EpS no puede pensarse por fuera de las fuerzas sociales que la determinan. Es necesario entonces ir más allá de las prácticas pedagógicas para analizar los discursos pedagógicos en el campo de la EpS y su relación con fuerzas estructurales, socioeconómicas, políticas y culturales que desempeñan un papel fundamental en la configuración de las prácticas pedagógicas.

Finalmente, habrá que reiterar la importancia de que la transformación de las prácticas pedagógicas corresponde también a un asunto de transformación del educador, lo que demanda procesos arduos de reflexión y esfuerzo continuado. Siguiendo a Freire, puede entenderse que la coherencia no es un punto de partida, sino un faro que orienta la acción pedagógica.

\section{Agradecimientos}

Esta publicación es resultado de una investigación realizada con el apoyo financiero de la Estrategia de Sostenibilidad del Comité para el Desarrollo de la Investigación (CODI) de la Universidad de Antioquia.

\section{Referencias bibliográficas}

1. Serrano M. Educación para la salud y participación comunitaria. Madrid: Díaz de Santos; 1990.

2. Nutbeam D. Health literacy as a public health goal: a challenge for contemporary health education and communication strategies into the 21st century. Health Promotion International. 2006;15(3):259-67.

3. Alzate Y. Desde la educación para la salud hacia la pedagogía de la educación alimentaria y nutricional. Perspectivas en Nutrición Humana. 2006;16(2):21-40.

4. López V. Marco conceptual de educación para la salud. En: Frías A. Salud pública y educación para la salud. Barcelona: Masson; 2000. p. 1-3.

5. Peñaranda F, Giraldo L, Barrera LH. La enseñanza de la educación para la salud: ¿una confrontación a la teoría y la práctica de la salud pública como disciplina? Rev Fac Nac Salud Pública 2015;33(3):353-360. doi: 10.17533/ udea.rfnsp.v33n3a04.

6. Díaz P, Peñaranda F, Cristancho S, Caicedo N, Garcés M, Alzate T, et al. Educación para la salud: perspectivas y experiencias de educación superior en ciencias de la salud, Medellín, Colombia. Rev Fac Nac Salud Pública. 2010;28(3):221-30.

7. Casas M. Educación para la salud: aspectos metodológicos. Educ Med Salud. 1991;25(2):194-202. 
8. Galeano M, Vélez O. Estado del arte en investigación cualitativa. Medellín: Universidad de Antioquia; 2002.

9. Plataforma temática conflicto urbano y jóvenes. Estado del arte conflicto urbano y jóvenes. Bogotá: CIVIS-ASDI; 2005.

10. Galeano M. Estrategias de investigación social cualitativa. El giro en la mirada. Medellín: La Carreta; 2012.

11. Álvarez C, González E. Lecciones de didáctica general. Medellín: Cooperativa Editorial Magisterio; 2002.

12. Barbosa J, Barbosa J, Rodríguez M. Revisión y análisis documental para estado del arte: una propuesta metodológica desde el contexto de la sistematización de experiencias educativas. Investig Bibl. 2013;27(61):83-105.

13. Rojas S. El estado del arte como estrategia de formación en la investigación. Studiositas (Bogotá). 2007;2(3):5-14.

14. Figueiredo M, Rodrigues-Neto J, Leite M. Modelos aplicados às atividades de educação em saúde. Rev Bras Enferm. 2010;63(1):117-21.

15. Pereira M, Padrón M, González L, Hernández T, Robainas R. Educación para la salud integral del adolescente a través de promotores pares. Rev Méd Electrón [Internet]. 2011; 33(3): 349-59.

16. Coscrato G, Bueno S. Concepts of health education by public health nurses. Rev Esc Enferm USP. 2013; 47(3):707-13.

17. González S, Giraldo M, Varela J, Peña E, Giraldo J, Orozco J. A family-focused oral-health toy library: towards a new direction in oral health education. Fac Odontol Univ Antioq. 2012;23(2):306-20.

18. Câmara A, Melo V, Gomes M, Pena B, Silva A, Oliveira K, et al. Percepção do processo saúde-doença: significados e valores da educação em saúde. Rev Brasileira de Educação Médica. 2012;36(supl. 1):40-50.

19. Agostoni C. Popular health education and propaganda in times of peace and war in Mexico City, 1890s-1920s. American Journal of Public Health. 2006;96(1):52-61.

20. Patino D, Miranda B, Ferreira B. Educação em saúde: percepção dos enfermeiros da atenção básica em Uberaba (MG). Ciência \& Saúde Coletiva. 2011;16(supl. 1):1547-54.

21. Ramírez N, Díaz M, Reyes P, Cueca O. Educación lúdica: una opción dentro de la educación ambiental en salud. Seguimiento de una experiencia rural colombiana sobre las geohelmintiasis. Rev Fac Med. 2011;19(1):23-36.

22. Almeida A, Soares C. Health education: analysis of its teaching in undergraduate nursing courses. Rev Latino-Am Enfermagem. 2011;19(3):614-21.

23. Vila A, Vila V. Trends of knowledge production in health education in Brazil. Rev Latino-am Enfermagem. 2007;15(6):1177-83.

24. Villarin A. El cuidado en salud y la educación salubrista como promoción de competencias humanas y autonomía. Salud Uninorte. 2008;24(2):341-50.

25. Sousa L, Torres C, Costa P, Bezerra A. Práticas de educação em saúde no Brasil: a atuação da enfermagem. Rev Enferm UERJ. 2010;18(1)55-60.

26. Salci M, Maceno P, Rozza S, Silva D, Boehs A, Heidemann I. Health education and its theoretical perspectives: a few reflections. Text Context Nursing. 2013;22(1):224-30.

27. Peñaranda F, Torres J, Bastidas M, Escobar G, Arango A, Pérez F. Praxis as a foundation for an alternative health education: an action research study in the Growth and Development Monitoring Program in Medellín, Colombia. Interface-Comunic Saude Educ. 2011;15(39):997-1008.

28. Días A, Carvalho C. Tendencias de la producción del conocimiento en la educación en la salud en Brasil. Rev Latino-am Enfermagem [Internet]. 2007 [citado 2014 jun. 15];15(6). Disponible en: http://www.scielo.br/pd f/rlae/v15n6/es_18.pdf

29. Díaz P. Theoretical conceptions on the theory on health education. Systematic review. Invest Educ Enferm. 2012;30(3):378-89.

30. Patino D, Miranda B, Ferreira B. Educação em saúde: percepção dos enfermeiros da atenção básica em Uberaba (MG). Ciência \& Saúde Coletiva. 2011;16(supl. 1):1547-54.

31. Escobar M, Aguirre L, Díaz V, León L, Moreno P, Soto S. Educación para la salud en una empresa social del Estado. Hacia la Promoción de la Salud. 2009;14(1):54-67. 
32. Arroyo H. Promoción de la salud y educación para la salud: paradigma integrador o el debate histórico de la inclusión o la exclusión. En: IV Conferencia Latinoamericana de Promoción de la Salud y Educación para la Salud; Medellín, 4-7 nov., 2009. Medellín: UIPES, Facultad Nacional de Salud Pública Universidad de Antioquia; 2009.

33. Cardacci D. Invisibilización de la educación para la salud. ¿estamos equivocándonos? En: IV Conferencia Latinoamericana de Promoción de la Salud y Educación para la Salud; Medellín, 4-7 nov., 2009. Medellín: UIPES, Facultad Nacional de Salud Pública Universidad de Antioquia; 2009.

34. Vélez G, Schwabe M. Educación permanente en la salud. Reflexiones en la perspectiva de la integralidad. Revista Cubana de Enfermería. 2010;26(4):667-79.

35. Merhy E, Feuerwerker L, Ceccim R. Educación permanente en salud: una estrategia para intervenir en la micropolítica del trabajo en salud. Salud Colectiva. 2006;2(2):147-60.

36. Stotz, E. Enfoques sobre educação popular e saúde. En: Ministério da Saúde-Secretaria de Gestão Estratégica e Participativa- Departamento de Apoio à Gestão Participativa. Caderno de Educação Popular e Saúde. Brasilia: Ministério da Saúde; 2007. p. 46-57.

37. Ocampo E. Corrientes pedagógicas para iniciar la aproximación [Internet] [citado 2005 ene. 25]. Disponible en: https://www.cinde.org.co/maestriamedellin/modulo\%20Corrientes\%20pedagogicas.pdf

38. Freire P. Pedagogía del oprimido. 14. ${ }^{a}$ edición. Buenos Aires: Siglo XXI; 1975.

39. Salci1 M, Maceno P, Rozza S, Vieira da Silva D, Boehs A, Heidemann I. Health education and its theoretical perspectives: a few reflections. Text Context Nursing. 2013;22(1):224-30.

40. Salleras L. Educación sanitaria: principios, métodos y aplicaciones. Madrid: Díaz de Santos; 1985.

41. Greene W, Simons-Morton B. Educación para la salud. México: Interamericana-McGraw-Hill; 1988.

42. Gazzinelli M, Gazzinelli A, Dos Reis D, De Mattos C. Educação em saúde: conhecimentos, representações e experiencias da doença. Cad Saúde Pública. 2005;21(1):200-6.

43. Abadía C, Oviedo D. Itinerarios burocráticos de la salud en Colombia: la burocracia neoliberal, su estado y la ciudadanía en salud. Rev Gerenc Polit. Salud. 2010;9(18):86-102.

44. Bourdieu P. Capital cultural, escuela y espacio social. México D. F.: Siglo XXI; 1998.

\section{Notas}

1 Educación para la salud es el término epistemológico que ocupa esta investigación; sin embargo, no es un término reconocido como descriptor de las ciencias de salud (término DeCS, como sí lo es el término educación en salud que aparece como palabra clave.

\section{Licencia Creative Commons CC BY 4.0}

Para citar este artículo: López Ríos JM, Molina Berrío DP, Peñaranda Correa F. Visibilidad y tendencias teóricas de la educación para la salud en cuatro países de América Latina, 2003-2013. Rev. Gerenc. Polit. Salud. 2018;17(35). https://doi.org/10.11144/Javeriana.rgps17-35.vtte 